\title{
Attosecond x-ray probing of laser-induced electron rescattering in atoms
}

\author{
Li Zhang (张丽) ${ }^{1}$ and Xinhua Xie (谢新华) $\odot^{2, *}$ \\ ${ }^{1}$ Communications and Electronic Information Department, Shanghai Vocational College of Science and Technology, \\ Shanghai 201800, China \\ ${ }^{2}$ SwissFEL, Paul Scherrer Institute, 5232 Villigen PSI, Switzerland
}

(Received 16 June 2020; accepted 3 August 2020; published 31 August 2020)

\begin{abstract}
We propose an attosecond time-domain spectroscopy to study laser-induced electron rescattering in atoms by using single photoionization with an attosecond x-ray pulse. From the time-frequency analysis of the time-domain signal, we obtained simultaneously the subcycle time and energy information of the rescattering electron wave packet during the interaction of a helium-like atom with a strong laser field. The obtained time and energy distributions nicely agree with the electron rescattering picture from the analysis based on the high-order harmonic response by the dipole acceleration and classical trajectory calculations. Contributions and interference from multiple rescattering can be unambiguously identified in the time and energy distributions of rescattering electrons. Since the observable of $\mathrm{x}$-ray single photoionization yield is proportional to the electron density near the nucleus, the proposed time-domain spectroscopy can in general be applied to studies of other light-induced linear and nonlinear polarization effects in atoms and molecules.
\end{abstract}

DOI: 10.1103/PhysRevResearch.2.033339

\section{INTRODUCTION}

Light-induced polarization is fundamental in most lightmatter interactions, especially nonlinear processes [1]. The origin of light-induced polarization is dominated by the response of valence electrons in the light field [1,2]. Valence electron dynamics in atoms and molecules is generally on the attosecond timescale; therefore, methodologies with attosecond temporal resolution are required to access such ultrafast dynamics $[3,4]$. When the light field is weak, the response of valence electrons in atoms and molecules is adiabatic and the induced polarization is linear to the light field strength. When atoms and molecules interact with a strong laser field, the response can be nonadiabatic and the induced polarization becomes highly nonlinear because of the high intensity of the laser pulse [5]. When the laser field becomes equivalently strong as the Coulomb force of an atom or a molecule, valence electrons can be removed from the bound system through tunneling or barrier-suppressed ionization, which leads to many fascinating strong-field processes ranging from photon emission through high-order harmonic generation (HHG) to electron emission through nonsequential double ionization [6-10]. In the laser-driven HHG process, the liberated electron is accelerated in the laser field and then driven back and recombines with its parent ion by emitting an extreme ultraviolet or x-ray photon which carries the energy loss of the recombined electron $[11,12]$. Depending on the shape of

\footnotetext{
*Corresponding author: xinhua.xie@psi.ch

Published by the American Physical Society under the terms of the Creative Commons Attribution 4.0 International license. Further distribution of this work must maintain attribution to the author(s) and the published article's title, journal citation, and DOI.
}

the driven field, isolated attosecond pulses or attosecond pulse trains can be produced, which can be used for time-resolved studies of ultrafast processes on the attosecond timescale [13-15]. Not only attosecond pulses but also attosecond electron bursts generated in the laser field can be applied to study ultrafast dynamics of atoms and molecules though photoelectron spectroscopy, based on electron wave-packet interference and electron diffraction on its parent ion [16-24].

Electron rescattering is essential to HHG and laser-induced electron interference and diffraction [11,12,16-18,25], and therefore plays a critical role in attosecond sciences [15]. The relation between the rescattering energy and time is the fundamental of attosecond methodologies using highorder harmonic spectroscopy and above-threshold ionization photoelectron spectroscopy [15]. Theoretical and numerical studies revealed the relation between rescattering energy and time using the dipole acceleration induced by the laser field $[11,12]$. Window Fourier transform of the dipole acceleration over interaction time provides direct access to the subcycle time and energy distribution of rescattering electrons. However, so far in experiments with the developed highorder harmonic spectroscopy and photoelectron spectroscopy could not directly provide the time and energy information of the electron rescattering process simultaneously. Highorder harmonic spectroscopy is based on the measurement of high-order harmonic properties, which suffers from the macroscopic propagation effect. The macroscopic effect can severely modify the spectrum shape: e.g., contributions from long electron trajectories and multiple rescattering can be in general strongly suppressed due to phase mismatching [26-29]. On the other hand, spectroscopies using photoelectron momentum or energy distributions have a common disadvantage. The measured electron momentum or energy distribution is a result of signal integration over the interaction time of the whole laser pulse, and therefore, the contributions 


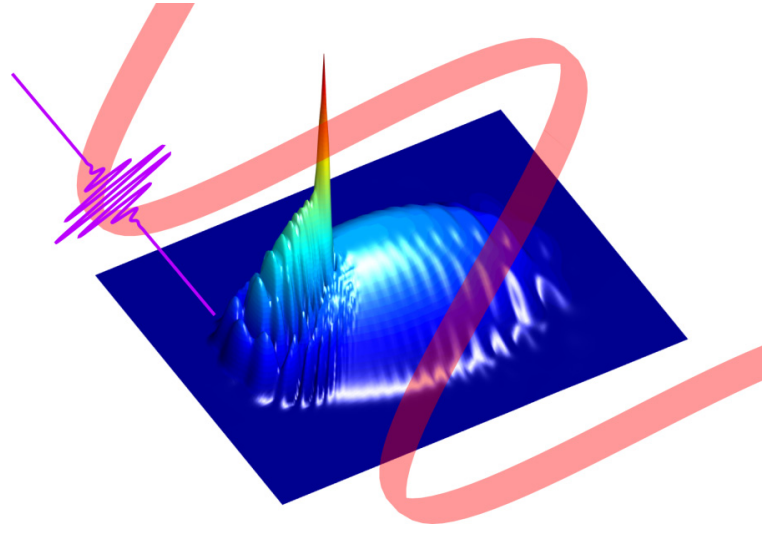

FIG. 1. A schematic view of x-ray probing of laser-induced nonlinear polarization of an atom. The surface plot illustrates a snapshot of an atomic electron wave packet from the numerical simulation. The ground state of the atom is shown as a sharp peak in the middle. The electron wave packet on the left side presents the ionizing electron wave packet from the atom, while the big hump on the right side exhibits the rescattering electron wave packet driving toward the atom by the laser field. A movie of electron density evolution in the laser field can be found in the Supplemental Materials [31].

of different ionization time are overlapped in the measured distribution and the disentanglement of different contributions is not trivial [30].

In this work, we propose an attosecond time-domain spectroscopy with $\mathrm{x}$-ray pulses to measure the laser-induced nonlinear polarization in atoms which allows us to get direct access to the time and energy information of the electron rescattering process simultaneously. In this method, using single photoionization yield as the observable, we exploit an attosecond $\mathrm{x}$-ray pulse to probe the nonlinear response of atoms in a strong near-infrared (NIR) laser pulse, which is schematized in Fig. 1.

\section{METHOD AND SIMULATIONS}

When an atom interacts with an external laser field, the valence electron wave packet is driven by the laser field and electron rescattering happens during the interaction (see the movie of the atomic electron wave packet driven by the laser field in the Supplemental Materials [31]). A delayed x-ray attosecond pulse is used to probe the distorted electron wave packet by the laser field. A previous theoretical study reveals that X-ray single photoionization yield is proportional to electron density near nucleus during the laser interaction with atoms [32]. It provides the possibility of using x-ray photoionization yield as a measure for probing electron polarization of atoms in a laser field. In our approach, the time-frequency analysis of the x-ray photoionization yield explicitly unveils the time and energy distribution of rescattering electrons during the interaction with a laser field.

To numerically simulate the proposed experiment, we solved the two-dimensional time-dependent Schrödinger equation (TDSE) with the single-active-electron approximation in velocity gauge. The pseudospectral method is employed for the propagation of the electron wave function in time within a spatial box of $[-600,600]$ a.u. in both directions. We applied a step-size-adapted fourthorder Runge-Kutta method to control the numerical errors during the electron wave function evolution in the external field $[33,34]$. In the simulations, we used a heliumlike atomic system which has a ground-state energy of -0.9 a.u. with a screened Coulomb potential $V(x, y)=$ $-1 / \sqrt{x^{2}+y^{2}+0.264}$. A linearly polarized laser and $\mathrm{x}$-ray pulse along the $x$ coordinate are applied in the simulations. The vector potentials of the X-ray $(X)$ and laser $(L)$ pulses are defined as $A_{F}(t)=\frac{\mathcal{E}_{F}}{\omega_{F}} A_{F 0}\left(t-t_{F}\right) \sin \left[\omega_{F}\left(t-t_{F}\right)\right]$ with $F=X, L$, where $\mathcal{E}_{F}$ and $\omega_{F}$ are the peak electric field and the center frequency. $t_{F}$ is the peak position of the pulse, which is used to control the time delay between the two pulses. $A_{F}(-\infty)=A_{F}(+\infty)=0$ is ensured to exclude any unphysical dc component in the pulses. In the simulations, a super-Gaussian envelope $A_{L 0}(t)=\exp \left(-2 \ln 2 \frac{t^{12}}{\tau_{L}^{12}}\right)$ is used for the laser pulse with center wavelength of $800 \mathrm{~nm} . \tau_{L}$ is chosen to $5 \mathrm{fs}$ such that the laser pulse has a flattop shape with three and a half optical cycles in the plateau. A Gaussian envelope $A_{X 0}(t)=\exp \left(-2 \ln 2 \frac{t^{2}}{\tau_{X}^{2}}\right)$ is used for the probing $x$-ray pulse with a photon energy of $400 \mathrm{eV}$ and a pulse duration full width at the half maximum (FWHM) $\tau_{X}$ of 20 as.

We performed simulations with NIR pumping pulses of peak intensity from $10^{14}$ to $10^{15} \mathrm{~W} / \mathrm{cm}^{2}$ and chose a peak intensity of $5 \times 10^{14} \mathrm{~W} / \mathrm{cm}^{2}$ to demonstrate our proposed method. According to the Keldysh parameter $\gamma=0.64$ [35], the laser-induced ionization of the helium-like atom is in the tunneling regime. The probing $x$-ray pulse has a peak intensity of $5 \times 10^{13} \mathrm{~W} / \mathrm{cm}^{2}$ to induce single photoionization. To simulate the pump-probe experiments, we fixed the NIR pulse and scanned the delay between the two pulses by varying $t_{X}$ with a step size of 6.67 as.

\section{RESULTS AND DISCUSSIONS}

\section{A. X-ray photoionization yield over time delay}

In the simulations, we define the $\mathrm{x}$-ray photoionization yield as a function of time delay $(\tau)$ by the difference between the ionization yield of the simulations with both pulses and with the NIR laser pulse only, which is calculated as

$$
\eta_{X}(\tau)=\left|\left\langle\Psi_{X}(\tau) \mid \Psi_{X}(\tau)\right\rangle\right|^{2},
$$

where $\Psi_{X}$ is the additional wave function amplitude due to the $\mathrm{X}$-ray pulse

$$
\left|\Psi_{X}(\tau)\right\rangle=\left(1-\left|\Psi_{L}(\tau)\right\rangle\left\langle\Psi_{L}(\tau)\right|\right)\left|\Psi_{L X}(\tau)\right\rangle
$$

with $\Psi_{L X}(\tau)$ and $\Psi_{L}(\tau)$ being the electron wave functions calculated with both pulses and only the laser pulse. The norm-squared of the additional wave function amplitude $\Psi_{X}$ gives us the photoelectron yield produced by the x-ray pulse. All time-dependent wave functions are obtained from results of solving the two-dimensional TDSE. The simulated x-ray photoionization yield is shown in Fig. 2(a) as a function of the time delay between the $\mathrm{x}$-ray and the NIR pulses. The $\mathrm{X}$-ray ionization yield shows a sinusoidal modulation with fine structures over the time delay [inset of Fig. 2(a)]. There are three types of features in the signal: (1) stepwise structure from 1 down to 0.99 , which is caused by the tunneling ioniza- 

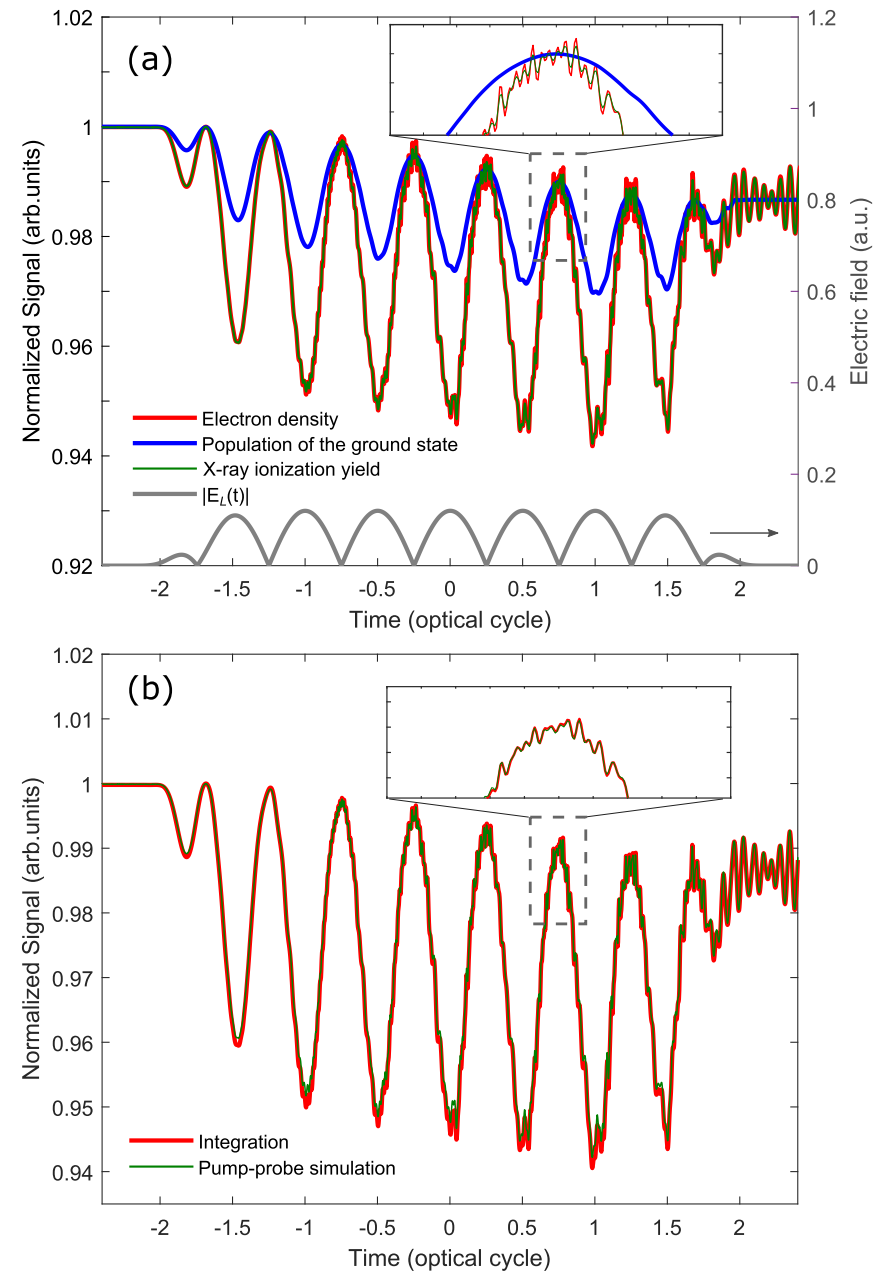

FIG. 2. (a) Normalized x-ray ionization yield (from the pumpprobe simulation), ground state population, and normalized electron density near origin as a function of the time (delay) for the helium-like atom in a linear laser pulse with a peak intensity of $5 \times 10^{14} \mathrm{~W} / \mathrm{cm}^{2}$. (b) Comparison of normalized $\mathrm{x}$-ray ionization yields from the pump-probe simulation and direct integration. The insets are used to show the agreement of the fast-oscillating signals. The corresponding electric fields are plotted with gray lines in panel (a) with the $y$ axis on the right side.

tion by the NIR pulse; (2) strong oscillation coinciding with the laser field which corresponds to adiabatic distortion by the NIR pulse; (3) fast and weak oscillations which originate from nonlinear responses due to nonadiabatic distortion induced by the NIR pulse. Note that the oscillation of the $x$-ray photoionization yield at the end and after the laser pulse originates from the quantum beating between the ground and excited states $[32,36]$.

In quantum theory, the photoionization cross section $(\sigma)$ by an X-ray pulse is defined by the transition strength between the initial bound state and the final continuum state [37]

$$
\sigma_{X} \propto\left|\left\langle k|p| \Psi_{i}\right\rangle\right|^{2}
$$

with $p$ being the momentum operator and $\langle k|$ being the continuum state. Here we only considered single photoionization induced by the $\mathrm{x}$-ray pulse and ignored any multiphoton processes which involved more $\mathrm{x}$-ray and NIR photons. Because of energy conservation, the momentum of the free electron $k_{ \pm}= \pm \sqrt{2\left(\omega_{X}-I_{p}\right)}$. Thus, with Eq. (3) we analytically obtained the x-ray single-photon ionization yield $\eta(\tau)$ with the integration form in two-dimensional Cartesian coordinate for linearly polarized $\mathrm{x}$-ray photon along $x$ direction with a time delay of $\tau$ as

$$
\eta_{X}(\tau) \propto \sum_{k_{ \pm}}\left|\iint p_{x} \Psi_{L}(\tau) e^{-i k_{ \pm} x} d x d y\right|^{2},
$$

where $\Psi_{L}(\tau)$ is the simulated time-dependent electron wave function with only the NIR laser pulse. To take the effect of the x-ray intensity profile into account, we convoluted the $\eta_{X}(\tau)$ with the same intensity profile of the x-ray pulse used in the pump-probe simulations. The convoluted $\eta_{X}(\tau)$ are plotted in Fig. 2(b), which closely agree with those from the pump-probe simulation. This finding indicates that the ionization by the $\mathrm{x}$-ray is dominated by single photoionization. Since the yields calculated from the two approaches are almost identical, we choose those from the pump-probe simulation for further analysis and discussion.

First of all, we need to understand the origin of the dependence of the x-ray ionization yield on the time delay. Intuitively, the x-ray ionization yield is determined by the population of the ground states of the system since the contribution from excitation states is minor due to much smaller photoionization cross sections. In Fig. 2(a), we present the population of the field-free ground state of the reference simulation without the $\mathrm{x}$-ray pulse. The population of the field-free ground state shows a similar dependence over time but with weaker modulations than the $\mathrm{x}$-ray ionization yield and no fast oscillations. On the other hand, the electron density near the nucleus with a radius of smaller than 2 a.u. is plotted as a function of time over the laser pulse, which shows a great agreement with the $\mathrm{x}$-ray probing signal including the fine structures [see the inset in Fig. 2(a)]. The deviations between the electron density and the x-ray ionization yield are from the averaging effect on the $\mathrm{x}$-ray ionization yield due to the pulse duration [36]. It leads to the information that the x-ray ionization yield is proportional to the electron density near the nucleus. Polarization of an atom is defined as the electron displacement away from the ground state, and therefore the electron density near the atom is a good quantity to present the strength of the polarization.

\section{B. Time-frequency analysis of time-domain signals}

To get the time and energy information contained in the simulated time-domain signals, we performed timefrequency analysis of them using the window Fourier transform with a time window width of 400 as. The timefrequency structure of the time-domain $x$-ray ionization signal is depicted in Fig. 3(a). Note that a spectral filter is applied to the time-frequency distribution to get rid of the effect of the pulse envelope, which will be discussed later. The structures in the distribution show a clear dependence of the frequency over the time delay. In the distribution, there is a clean curved structure for the first half optical cycle and such structure is repeated every half optical cycle. From the second half optical cycle on, there are more complicated structures under the curved structure. Such structures are 

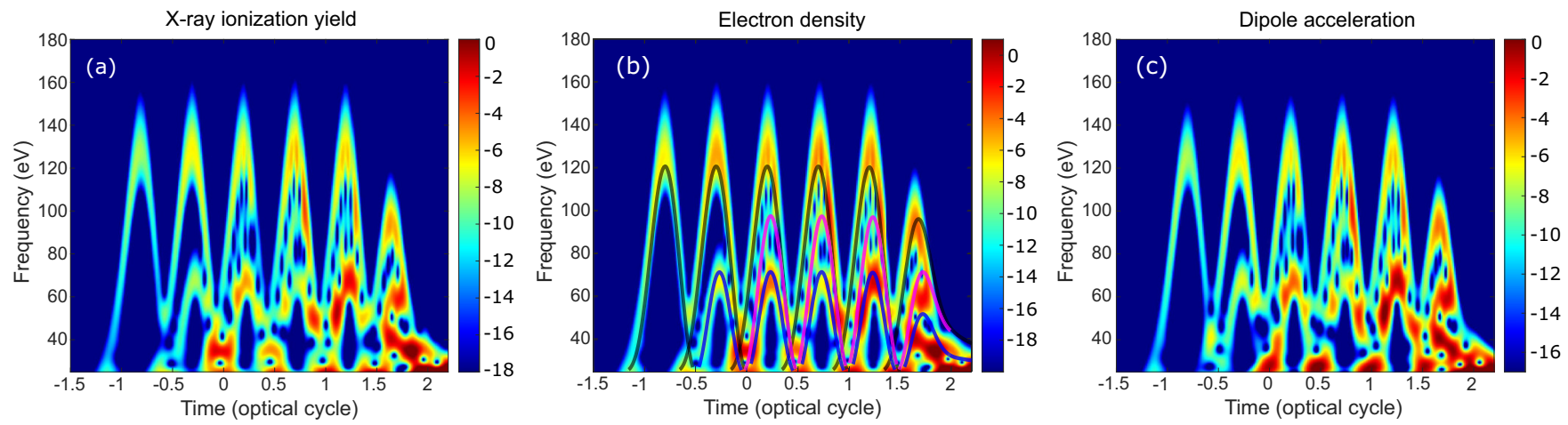

FIG. 3. Time and energy distributions from window Fourier transform of (a) x-ray ionization yield, (b) electron density, and (c) dipole acceleration over time (delay). The three lines in (b) represent the first (gray), second (magenta), and third (blue) electron rescattering energies added by the ionization potential over time from classical trajectory calculations.

similar to the well-known structures in the time-frequency structure of HHG signals [38]. To make a close comparison, we calculated the HHG response by calculating the dipole acceleration from the simulated electron wave function [12]. The time-frequency structure of the dipole acceleration is illustrated in Fig. 3(c), which are near "identical" to those from the time-domain $x$-ray ionization yield in Fig. 3(a). The time-frequency structure clearly shows the electron rescattering process over time during the laser pulse: when electrons rescatter with which energy. Nevertheless, the time-frequency distribution of the electron density near the nucleus is also illustrated in Fig. 3(b), which shows exactly the same as that from the x-ray ionization yield. For electron rescattering, the released electron needs to be driven back to the origin to scattering with its parent ion. Therefore, the information of the rescattering electron is contained in the electron density near the nucleus.

To understand the structures in the time-frequency distributions, classical trajectory simulations were carried out by solving the Newtonian equation of a free electron in the NIR laser field to get the electron rescattering energy over the rescattering time. In the simulations, the effect of Coulomb potential is neglected and the rescattering energy is calculated when an electron returns to the origin. During the laser pulse, an electron can possibly return to the origin multiple times, which is also referred as multiple rescattering. We present the recombination energy $\left(E_{\text {res }}+I_{p}\right)$ of the first, second, and third rescatterings as different colored lines in Fig. 3(b). The first rescattering lines (gray) closely fit with the repeated timefrequency structures of each half optical cycle. On the other hand, the second and third rescatterings happen with lower rescattering energies, which fit into the complicated structures under the first rescattering curves. The complicated structures are not shown as a continuous line shape, which is due to interference of multiple rescattering, e.g., between electron wave packet released at different times but rescattering with the same energy at the same time. Such interference can lead to the enhancement or suppression of the signal, e.g., in the time window between 0 and 0.5 optical cycle, the classic results show that the first rescattering in the long trajectory side overlaps with the second rescattering from the previous half optical cycle and the overlapping leads to the suppression of the signal in the energy range from 60 to $90 \mathrm{eV}$. Similar overlapping happens after a half optical cycle; however, the interference leads to the enhancement of the signal in the same energy range [38]. Such interference between different rescattering events can be exploited to coherent control of HHG.

\section{Requirement of x-ray pulse duration}

In the end, we check the requirement on x-ray pulse duration, a key parameter for the time-domain spectroscopy. For single photoionization, the ionization yield is proportional to the intensity of the x-ray pulse, and therefore, the effect of pulse duration is applied to the measured signal through convolution with the intensity profile of the pulse. In the Fouriertransformed frequency domain, the effect is contributed as a low-pass filter, which is the Fourier transform of the $\mathrm{x}$-ray pulse intensity profile over time. Figure 4 presents the electric field and envelope of the x-ray pulse and the corresponding low-pass filter. With the pulse duration $\tau_{X}=20$ as and a Gaussian profile, the low-pass filter is also a Gaussian function with the half width at half maximum of $4 \ln 2 / \tau_{X}=91.3 \mathrm{eV}$, which
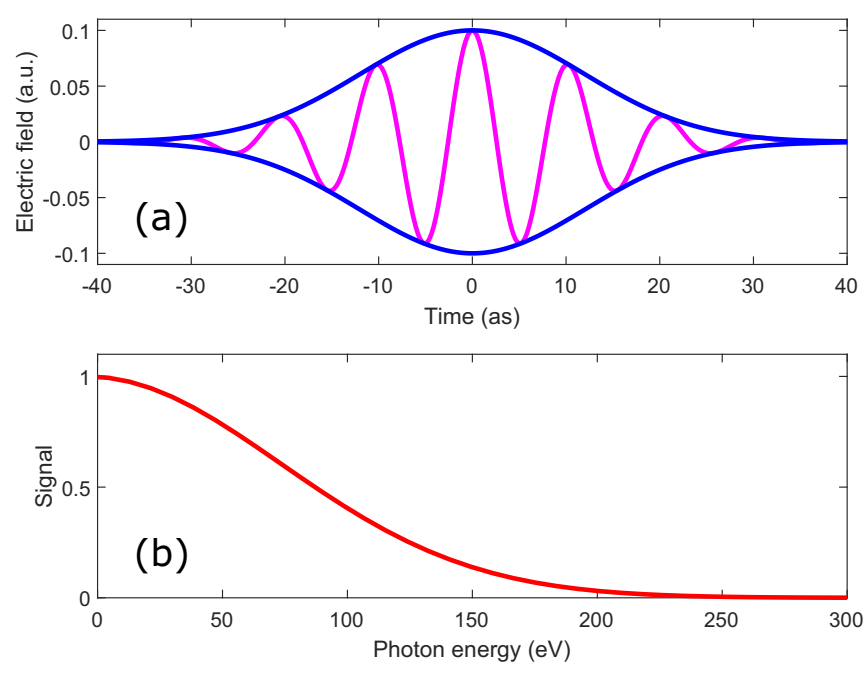

FIG. 4. (a) The electric field of the attosecond x-ray pulse with a Gaussian envelope. (b) Low-pass filter in the frequency domain corresponding to the intensity envelope of the $\mathrm{x}$-ray pulse. 
is inversely proportional to the pulse duration. To resolve ultrafast electron dynamics, the choice of the parameters of the x-ray pulse is critical. To measure dynamics on the timescale corresponding to the recombination energy of $E_{\mathrm{rec}}$, the pulse duration of the $\mathrm{x}$-ray pulse should be at least $4 \sqrt{2 \ln 2} / E_{\text {rec }}$ if we consider the half width of the low-pass filter at $1 / e^{2}$ of the maximum. The pulse duration of the $\mathrm{x}$-ray pulse further limits the photon energy of the pulse since the pulse duration should be at least longer than one optical cycle of the pulse. For an experiment with targets of lower ionization potentials, the laser peak intensity can be much lower to induce a strong field process such that $\mathrm{x}$-ray pulses with much longer pulse durations and much lower photon energies can be exploited for the time-domain spectroscopy.

\section{CONCLUSION}

In conclusion, we proposed an attosecond time-domain spectroscopy with attosecond $\mathrm{x}$-ray pulses to study laserinduced ultrafast electron dynamics in atoms and molecules. Using the $\mathrm{x}$-ray single photoionization yield as the observable, the time-frequency analysis of the time-domain signal reveals simultaneously the time and energy information of rescattering electrons during the interaction of an atom with a strong laser field. The time and energy structure nicely agrees with the electron rescattering picture from the analysis based on the high-order harmonic response by the dipole acceleration and classical trajectory calculations. Contributions and interference from multiple rescattering can be explicitly identified in the acquired time and energy distribution, which allows further detailed studies on the impact of multiple rescattering on the rescattering related strong field processes. Since the observable of x-ray single photoionization yield is proportional to the electron density near the nucleus, the proposed time-domain spectroscopy can in general be applied to study the dynamics in any other light-induced linear and nonlinear polarization effects.

\section{ACKNOWLEDGMENTS}

This work was partially financed by the Austrian Science Fund (FWF) under P30465-N27, and the computational results presented have been achieved in part using the Vienna Scientific Cluster (VSC).
[1] R. W. Boyd, Nonlinear Optics (Academic Press, San Diego, 2019).

[2] R. Menzel, Photonics: Linear and Nonlinear Interactions of Laser Light and Matter (Springer, Berlin, 2007).

[3] E. Goulielmakis, Z.-H. Loh, A. Wirth, R. Santra, N. Rohringer, V. S. Yakovlev, S. Zherebtsov, T. Pfeifer, A. M. Azzeer, M. F. Kling, S. R. Leone, and F. Krausz, Nature (London) 466, 739 (2010).

[4] J. Itatani, J. Levesque, D. Zeidler, H. Niikura, H. Pépin, J. C. Kieffer, P. B. Corkum, and D. M. Villeneuve, Nature (London) 432, 867 (2004).

[5] T. Brabec and F. Krausz, Rev. Mod. Phys. 72, 545 (2000).

[6] A. McPherson, G. Gibson, H. Jara, U. Johann, T. S. Luk, I. A. McIntyre, K. Boyer, and C. K. Rhodes, J. Opt. Soc. Am B 4, 595 (1987)

[7] A. L'Huillier and P. Balcou, Phys. Rev. Lett. 70, 774 (1993).

[8] S. Larochelle, A. Talebpour, and S. L. Chin, J. Phys. B: At. Mol. Opt. Phys. 31, 1201 (1998).

[9] A. Staudte, C. Ruiz, M. Schöffler, S. Schössler, D. Zeidler, T. Weber, M. Meckel, D. M. Villeneuve, P. B. Corkum, A. Becker, and R. Dörner, Phys. Rev. Lett. 99, 263002 (2007).

[10] A. Rudenko, V. L. B. de Jesus, T. Ergler, K. Zrost, B. Feuerstein, C. D. Schröter, R. Moshammer, and J. Ullrich, Phys. Rev. Lett. 99, 263003 (2007).

[11] P. B. Corkum, Phys. Rev. Lett. 71, 1994 (1993).

[12] M. Lewenstein, P. Balcou, M. Y. Ivanov, A. L'Huillier, and P. B. Corkum, Phys. Rev. A 49, 2117 (1994).

[13] P. M. Paul, E. S. Toma, P. Breger, G. Mullot, F. Augé, P. Balcou, H. G. Muller, and P. Agostini, Science 292, 1689 (2001).

[14] A. Baltuška, T. Udem, M. Uiberacker, M. Hentschel, E. Goulielmakis, C. Gohle, R. Holzwarth, V. S. Yakovlev, A. Scrinzi, T. W. Hänsch, and F. Krausz, Nature (London) 421, 611 (2003).

[15] F. Krausz and M. Ivanov, Rev. Mod. Phys. 81, 163 (2009).
[16] R. Gopal, K. Simeonidis, R. Moshammer, T. Ergler, M. Dürr, M. Kurka, K.-U. Kühnel, S. Tschuch, C.-D. Schröter, D. Bauer, J. Ullrich, A. Rudenko, O. Herrwerth, T. Uphues, M. Schultze, E. Goulielmakis, M. Uiberacker, M. Lezius, and M. F. Kling, Phys. Rev. Lett. 103, 053001 (2009).

[17] Y. Huismans, A. Rouzée, A. Gijsbertsen, J. H. Jungmann, A. S. Smolkowska, P. S. W. M. Logman, F. Lépine, C. Cauchy, S. Zamith, T. Marchenko, J. M. Bakker, G. Berden, B. Redlich, A. F. G. van der Meer, H. G. Muller, W. Vermin, K. J. Schafer, M. Spanner, M. Y. Ivanov, O. Smirnova, D. Bauer, S. V. Popruzhenko, and M. J. J. Vrakking, Science 331, 61 (2011).

[18] X. Xie, S. Roither, D. Kartashov, E. Persson, D. G. Arbó, L. Zhang, S. Gräfe, M. S. Schöffler, J. Burgdörfer, A. Baltuška, and M. Kitzler, Phys. Rev. Lett. 108, 193004 (2012).

[19] X. Xie, Phys. Rev. Lett. 114, 173003 (2015).

[20] H. Niikura, F. Légaré, R. Hasbani, A. Bandrauk, M. Y. Ivanov, D. M. Villeneuve, and P. B. Corkum, Nature (London) 417, 917 (2002).

[21] S. N. Yurchenko, S. Patchkovskii, I. V. Litvinyuk, P. B. Corkum, and G. L. Yudin, Phys. Rev. Lett. 93, 223003 (2004).

[22] M. Spanner, O. Smirnova, P. B. Corkum, and M. Y. Ivanov, J. Phys. B: At. Mol. Opt. Phys. 37, L243 (2004).

[23] M. Meckel, D. Comtois, D. Zeidler, A. Staudte, D. Pavičić, H. C. Bandulet, H. Pépin, J. C. Kieffer, R. Dörner, D. M. Villeneuve, and P. B. Corkum, Science 320, 1478 (2008).

[24] K. Amini, M. Sclafani, T. Steinle, A.-T. Le, A. Sanchez, C. Müller, J. Steinmetzer, L. Yue, J. R. Martínez Saavedra, M. Hemmer, M. Lewenstein, R. Moshammer, T. Pfeifer, M. G. Pullen, J. Ullrich, B. Wolter, R. Moszynski, F. J. García de Abajo, C. D. Lin, S. Gräfe, and J. Biegert, Proc. Natl. Acad. Sci. USA 116, 8173 (2019).

[25] D. G. Arbó, K. L. Ishikawa, K. Schiessl, E. Persson, and J. Burgdörfer, Phys. Rev. A 81, 021403(R) (2010). 
[26] A. L'Huillier, K. J. Schafer, and K. C. Kulander, Phys. Rev. Lett. 66, 2200 (1991).

[27] P. Antoine, A. L'Huillier, and M. Lewenstein, Phys. Rev. Lett. 77, 1234 (1996).

[28] A. Rundquist, C. G. Durfee, Z. Chang, C. Herne, S. Backus, M. M. Murnane, and H. C. Kapteyn, Science 280, 1412 (1998).

[29] P. Salières and I. Christov, in Strong Field Laser Physics (Springer, Berlin, 2008), pp. 261-280.

[30] C. Hofmann, A. S. Landsman, and U. Keller, Appl. Sci.-Basel 8, 341 (2018).

[31] See Supplemental Material at http://link.aps.org/supplemental/ 10.1103/PhysRevResearch.2.033339 for the movie of the electron wave packet in a strong laser field.
[32] Y. Deng and X. Xie, Phys. Rev. A 91, 043414 (2015).

[33] X. Xie, G. Jordan, M. Wickenhauser, and A. Scrinzi, J. Mod. Opt. 54, 999 (2007).

[34] X. Xie, M. Wickenhauser, W. Boutu, H. Merdji, P. Salières, and A. Scrinzi, Phys. Rev. A 76, 023426 (2007).

[35] L. V. Keldysh, Sov. Phys. JETP 20, 1307 (1965).

[36] X. Xie, Ph.D. thesis, Technische Universität Wien, Vienna, Austria, 2007

[37] B. H. Bransden and C. J. Joachain, Physics of Atoms and Molecules, Second edition (Pearson Education, Harlow, England, 2003).

[38] C. Zhang, C. Xia, X. Jia, and X. Miao, Opt. Express 24, 20297 (2016). 